\title{
Nonalcoholic Fatty Liver Disease: A New Frontier for Hepatology in Bangladesh and a Call for Action to Combat
}

\author{
S ALAM $^{\mathrm{a}}$, MM HOSSAIN $^{\mathrm{b}}$, G AZAM $^{\mathrm{c}}$, G MUSTAFA $^{\mathrm{d}}$, M ALAM $^{\mathrm{e}}$, \\ A SAYEED $^{\text {f }}$, M KHAN', N AHMED ${ }^{\mathrm{h}}$
}

\begin{abstract}
Summary:
Nonalcoholic Fatty Liver Disease (NAFLD) is the condition where fat accumulates in liver without significant ingestion of alcohol. NAFLD has become one of the most common liver conditions throughout the world. At the dawn of the history of NAFLD it was thought that NAFLD is disease of obese individual but lean patients are increasingly detected to have NAFLD. It seems that insulin resistance is central to the pathogenesis of NAFLD. In addition, oxidative stress and cytokines are important contributing factors, resulting in steatosis and progressive liver damage in genetically susceptible individuals. NAFLD varies considerably by ethnic group and Bangladeshi ethnicity is an independent risk factor for NAFLD. Prevalence of NAFLD in general population of Bangladesh is $4-18.4 \%$, which jumps up to $49.8 \%$ in diabetic patients. With the changes in socioeconomic condition and life style, aetiology
\end{abstract}

Introduction:

Nonalcoholic fatty liver disease (NAFLD) is a condition characterized by excessive accumulation of lipid

a. Dr. Shahinul Alam, Associate Professor, Dept. of Hepatology, Bangabandhu Sheikh Mujib Medical University, Dhaka.

b. Dr. M Motahar Hossain, Culsultant, Gastroenterology and Hepatology, Square Hospital, Dhaka, Bangladesh.

c. Dr. Golam Azam, Assistant Professor of Gastroentestinal Liver and Pancreatic Disease, BIRDEM, Dhaka.

d. Dr. Golam Mustafa, Associate Professor, Dept. of Hepatology, Bangabandhu Sheikh Mujib Medical University, Dhaka.

e. Dr. Mahbubul Alam, Medical Officer, Dept. of Hepatology, Bangabandhu Sheikh Mujib Medical University, Dhaka.

f. Prof. Abu Sayeed, Professor of Medicine (Rtd.), Chittagong Medical College, Chittagong.

g. Prof. Mobin Khan, Professor and Chairman (Rtd), Dept. of Hepatology, Bangabandhu Sheikh Mujib Medical University, Shahbag, Dhaka.

h. Prof. Nooruddin Ahmad, Professor and Chieman, Dept. of Hepatology, Bangabandhu Sheikh Mujib Medical University, Dhaka.

Address of Correspon: Dr. Shahinul Alam, Associate Professor, Dept. of Hepatology, Bangabandhu Sheikh Mujib Medical University, Shahbag, Dhaka-1000, Bangladesh, Cell: 01973007173, E-mail: shahinul@yahoo.com

Received: 22 May 2016

Accepted: 30 Sept. 2017 of chronic liver disease is drifting from infectious to noninfectious diseases and the contribution of NAFLD is progressively increasing. Hepatitis $B$ and hepatitis Chave been the leading causes of mortality and morbidity from chronic liver disease in Bangladesh. But with increase in awareness and mass vaccination against $\mathrm{HBV}$, prevalence of both the diseases has been decreasing in the country. The most alarming feature is that there is a high prevalence of NASH among the NAFLD patients. NAFLD is emerging as the largest contributor of chronic liver disease in Bangladesh. This warrants the attention of health policy makers and clinicians to explore this frontier and combat it from right now.

Key words: Fatty liver, Bangladesh, Prevalence, Cirrhosis, Non-communicable disease.

(J Bangladesh Coll Phys Surg 2017; 35: 184-191)

(defined as the presence of lipid in $>5 \%$ of hepatocytes or a lipid content $>5 \%$ liver weight) ${ }^{1}$ in the liver in individuals, who consume little $(<20 \mathrm{~g}$ of alcohol/d) or no alcohol.1, ${ }^{2}$ It is the most common cause of chronic liver injury. ${ }^{3}$ When NAFLD is accompanied with liver cell injury and inflammation it is called nonalcoholic steatohepatitis (NASH)2. About 30\% NAFLD progress to NASH, if untreated it can be lead to fibrosis, cirrhosis or even hepatocellular carcinoma (HCC). ${ }^{4}$ Indeed, NAFLD is now recognized to be the aetiology in many cases previously labelled as cryptogenic cirrhosis. ${ }^{5}$ $\mathrm{HCC}$ is one of the most common cancers worldwide and its burden is the highest in South-East Asia. ${ }^{6}$ In the Asia-Pacific region the prevalence of NAFLD has increased remarkably over the years affecting up to $30 \%$ of the general population. ${ }^{7}$ Prevalence of NAFLD in adult population both in developing and developed Asian countries has been increasing, the figures vary from 9 to $30 \%$ in Japan, 5 to $24 \%$ in China, 5 to $28 \%$ in India, $18 \%$ in Korea, $30 \%$ in Malaysia and 5\% in Singapore. ${ }^{8}$ Metabolic syndrome, common in people from South Asia, is an important risk factor for NAFLD 
with Bangladeshi ethnicity being an important independent risk factor.3 NAFLD has become an important public health problem because of its high prevalence, potential to progress to severe liver disease and association with serious cardio-metabolic abnormalities including type-2 diabetes mellitus (T2DM), metabolic syndrome and coronary heart disease. Of particular concern, and with significant implications for future disease burden, is the increasing prevalence of NAFLD in children and young adults. Studies have reported a $3 \%$ prevalence of NAFLD in the general paediatric population, rising to $53 \%$ in obese children. ${ }^{9,}{ }^{10}$ With the increasing prevalence of obesity, diabetes, and metabolic syndrome in the general population, NAFLD has become the most common cause of chronic liver disease in the western countries as well as lower BMI areas such as in regions of Asia. Thus the nature and extent of the problem of NAFLD in the context of Bangladesh needs to be addressed seriously.

\section{Pathogenesis of NAFLD and diagnosis:}

The pathogenesis of NAFLD is still not fully elucidated. It seems that insulin resistance (IR) related to obesity is central to the pathogenesis of NAFLD (Figure - 1). In addition, oxidative stress and cytokines are important contributing factors, together resulting in steatosis and progressive liver damage in genetically susceptible individuals. Key histological components of NASH are steatosis, hepatocellular ballooning, and lobular inflammation. The degree of fibrosis on liver biopsy (stage) is predictive of the prognosis. Current evidences are in favor of the "multiple-hit hypothesis," for the pathogenesis of NAFLD where the first insult, or hit, to the liver occurs from insulin resistance. ${ }^{11}$ This causes increased levels of fatty acids that result in fatty infiltration of the liver, or steatosis. These hits continue and the increasing fatty acids eventually cause apoptosis, or cell death, of hepatocytes. The identities of the multiple "hits" are probably different in each patient and largely undefined at present. ${ }^{12}$ Sedentary life style, metabolic syndrome, diabetes mellitus, and dyslipidameia are risk factors of NAFLD in non-obese persons. $6^{13}$ Diabetes is also one of the main culprits in the progression to $\mathrm{NASH},{ }^{14}$ and liver biopsy is recommended in NAFLD patients who have diabetes and elevated GGT. ${ }^{14}$ Although elevated liver enzymes are consequences of NAFLD, ${ }^{13}$ male sex, raised ALT, high LDL cholesterol, and insulin resistance are significant and independent factors associated with the presence of NAFLD in T2DM subjects. ${ }^{15}$ Furthermore, South Asian populations may be more genetically susceptible via the inheritance of polymorphisms in apolipoprotein-3 that increase IR and cause NAFLD (Figure - 2). ${ }^{16}$

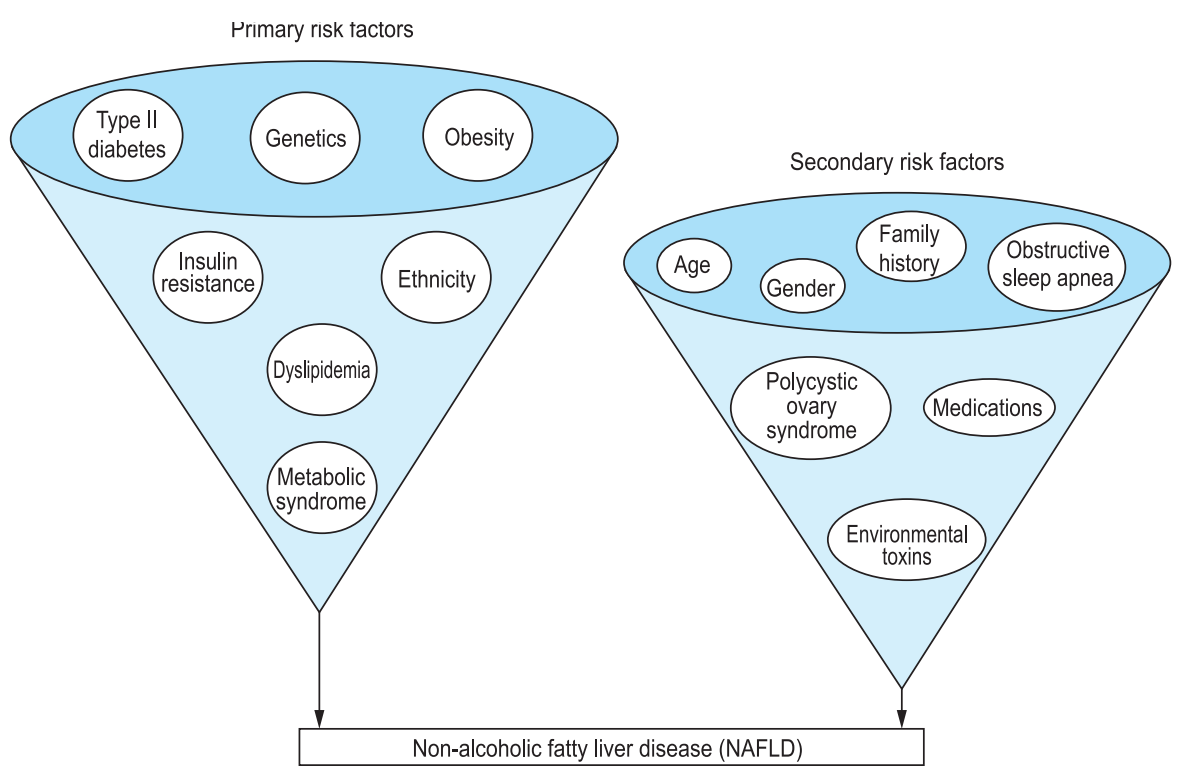

Fig.-1: Risk factors for NAFLD. 


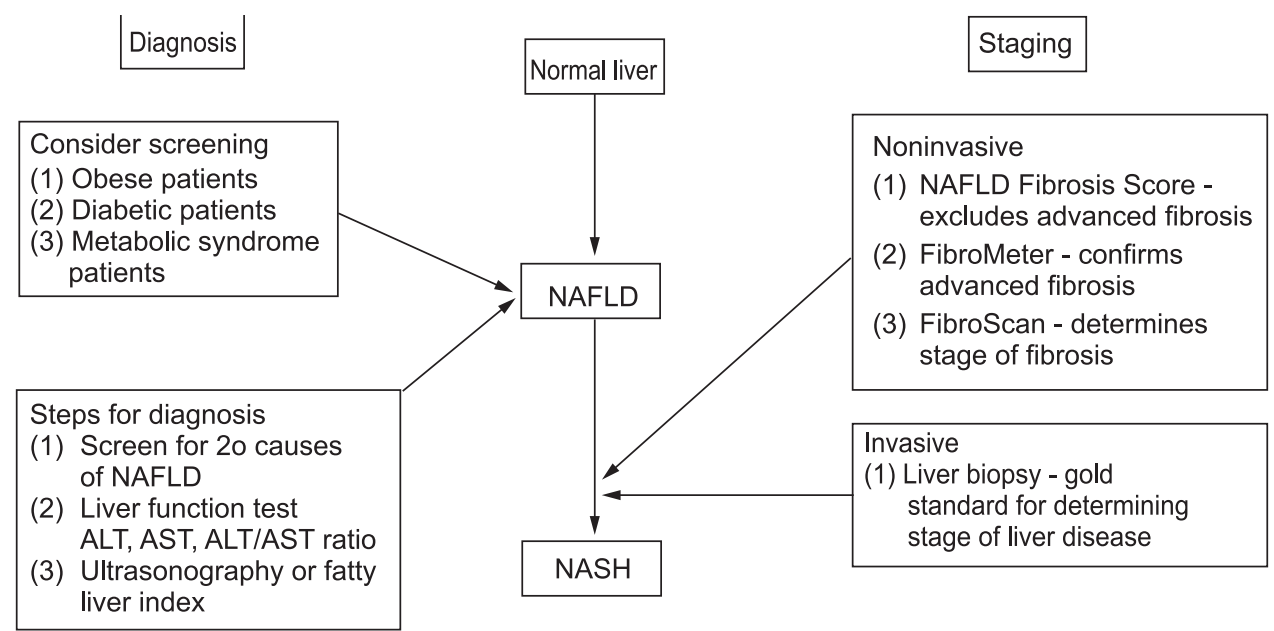

Fig.-2: Showing diagnosis steps and staging of NAFLD. 12

\section{Burden of NAFLD:}

It is increasingly apparent that NASH and NAFLD are not Western diseases. There is evolution of Westernstyle life among the Asian population and NASH has increasingly been diagnosed in several regions in Asia (Table- I) ${ }^{17}$ A study using the National Health and Nutrition Examination Survey (NHANES) found a 30\% rate of NAFLD in the United States between 2011 and $2012 .{ }^{18}$ NAFLD affects about 1 billion individuals worldwide and is the most common cause of chronic liver disease in Western countries. ${ }^{19}$ NASH is an increasingly common chronic liver disease with worldwide distribution that is closely associated with diabetes and obesity, which have both reached epidemic proportions (Figure -3). It is estimated that there are at least 1.46 billion obese adults worldwide. The Asia Pacific region is the most diverse and the most populous region in the world. Recent socioeconomic changes with increased affluence and changes in lifestyle have resulted in an emerging epidemic of non-communicable diseases such as T2DM and NAFLD. The prevalence of NAFLD in Asian Pacific countries now approximates that seen in Western countries, ${ }^{16}$ and is thus an emerging healthcare priority in Asia. This has a potential impact not only for the emerging liver disease burden in this region but also as a broader public health issue in view of the

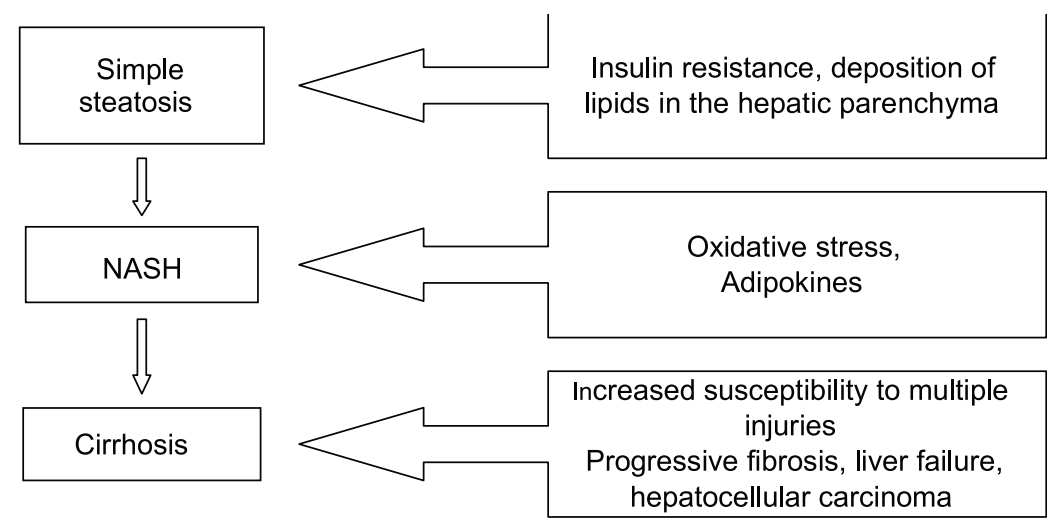

Fig.3: Progression of NAFLD 
association of NAFLD with the other metabolic syndrome-linked noncommunicable diseases - obesity, diabetes, and atherosclerotic cardiovascular disease. ${ }^{15}$

Most of the available epidemiological studies in NAFLD from Asia are ultrasound based and hence detect prevalence of hepatic steatosis alone initially, correlating it with anthropometric, biochemical, and demographic features of the population (Table 2). The community prevalence of NAFLD in South Asia and South East Asia ranges from 5- 30\% ${ }^{20}$ In India it varies from 5$28 \%$ in general population especially those who are undergoing healthy checkups. ${ }^{20}$

According to Zhang et al study, with the globally increasing prevalence, NAFLD becomes the predominant cause of chronic liver disease. 2 The global scientific research of NAFLD are increasing drastically. To sum up, 6356 articles were published in 994 different journals during 1986-2013. Starting from the late 1980s, the publication on NAFLD grew slowly and entered into a highly developing period in the 21 st century, especially in the last decade (Figure 4). Bibliometric results suggest that the obviously rapid growth of the articles in recent years appears to be associated with the accelerating incidence of NAFLD. In addition, epidemiology focusing on comparing different regions and population is attracting ever-growing attention.

\section{Burden of NAFLD in Bangladesh:}

Alazawi et al3 demonstrated that the prevalence of recorded NAFLD varies considerably by ethnic group and Bangladeshi ethnicity is an independent risk factor for NAFLD. Among Bangladeshis, there are high rates of T2DM and cardiovascular disease that may have a genetic basis. In that study in the UK, liver function tests were performed on 218,032 patients, of whom 31,627 had elevated serum transaminases. The prevalence of NAFLD was the most frequently recorded liver disease and was most common among Bangladeshi patients. In a multivariate analysis, independent risk factors for NAFLD included Bangladeshi ethnicity, diabetes, raised BMI, hypertension, and hypercholesterolaemia. Diagnosed NAFLD was significantly more prevalent among people of Bangladeshi ethnicity ( $1.8 \%$ of the adult population) than any other ethnic group, including other South Asian groups (Table 3 and Figure 5). The prevalence of NAFLD was significantly lower in the African and Caribbean ethnic groups. ${ }^{15}$

Prevalence of NAFLD in general population of Bangladesh has been estimated to vary from 4 to 18.4 $\%$, which jumps up to $49.8 \%$ in diabetic patients. ${ }^{21,22}$ Rahman et al in their rural population based study revealed prevalence of $18.4 \%$ with a higher prevalence of $59.4 \%$ in diabetic patients. In this study binary logistic

\section{Table-I}

\section{Prevalence of NAFLD among different areas of the word.}

\begin{tabular}{llc}
\multicolumn{2}{l}{ Prevalence of NAFLD among different areas of the word. } \\
Region & Population Studied & $\begin{array}{c}\text { Prevalence of NAFLD in these } \\
\text { populations (\%) }\end{array}$ \\
\hline USA & Pediatric population & $13-14$ \\
& General population & $27-34$ \\
& Morbid obesity & $75-92$ \\
& European-Americans & 33 \\
Europe & Hispanic-American & 45 \\
& African-American & 24 \\
Western countries & Pediatric Population & $2.6-10$ \\
& General Population & $20-30$ \\
Worldwide & Pediatric population & $20-40$ \\
Middle East & Obesity or diabetes & 75 \\
Far East & Morbid obesity & $90-95$ \\
Pakistan & Obese population & $40-90$ \\
& General population & $20-30$ \\
& General population & 15 \\
& General population & 18 \\
\hline
\end{tabular}


Table-II

\section{Showing prevalence of NAFLD among the Indian, Sri Lanka and Pakistani people}

\begin{tabular}{llcc} 
Country and place & Population & Sample size (n) & Prevalence of NAFL \\
\hline Mumbai, India & Selected population (railway colonies) & 1168 & $16.6 \%$ \\
West Bengal, India & General population (Rural) & 1911 & $167(8.7 \%)$ \\
Chennai, India & General population (Urban) & 541 & $173(32 \%)$ \\
Sri Lanka & General population (Urban) & 2985 & $974(32.6 \%)$ \\
Karachi, Pakistan & Tertiary care hospital & 952 & $129(13.6 \%)$ \\
\hline
\end{tabular}

Table-III

Showing Prevalence of NAFLD among different ethnic group living in UK.

\begin{tabular}{lccccccc} 
& Bangladeshi & Indian & Pakistani & White & African & Caribbean & Total \\
\hline $\mathrm{n}$ & 1678 & 325 & 245 & 1889 & 209 & 143 & 5250 \\
Prevalence $\%$ & 1.8 & 0.6 & 0.8 & 0.7 & 0.4 & 0.6 & 0.8 \\
\hline
\end{tabular}

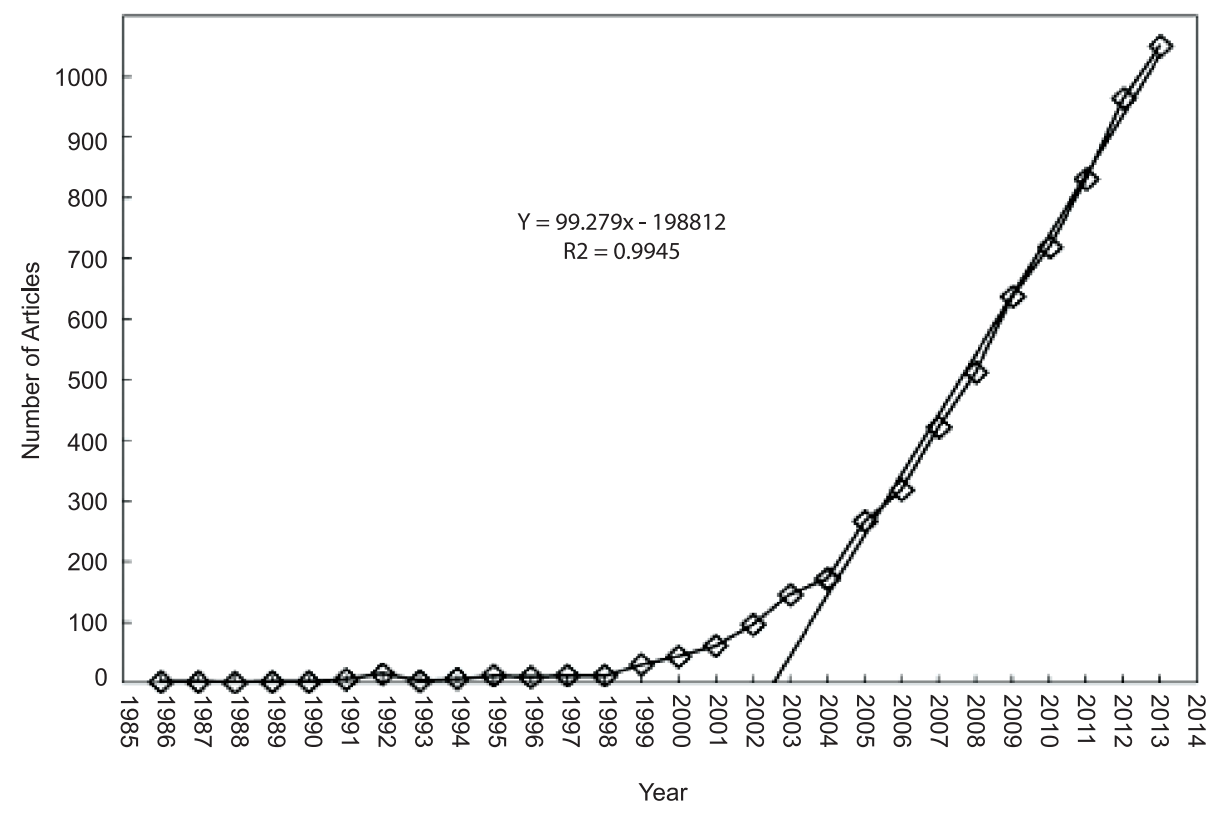

Fig.-4: Publication trend of article on NAFLD from 1986 to 2013.

regression analysis explored that diabetes, obesity $(\mathrm{BMI}>25)$, increased waist circumference and hypertriglyceridemia are independent risk factors for development of NAFLD. ${ }^{22}$ This is the $2^{\text {nd }}$ commonest cause of hepatology out-patient consultation in the country after chronic hepatitis B. ${ }^{23}$ These data are in concordance with prevalence of neighboring countries. $^{24-2526}$

\section{Unique character of NAFLD in Bangladesh:}

Though NAFLD appears to be a disease of the obese, in Bangladesh non-obese populations are also affected and the severity is also similar to that found in obese population. ${ }^{22,27,28}$ Though NAFLD is a male predominating disease in Europe and USA, it appears to affect Bangladeshi females more than males. Social 


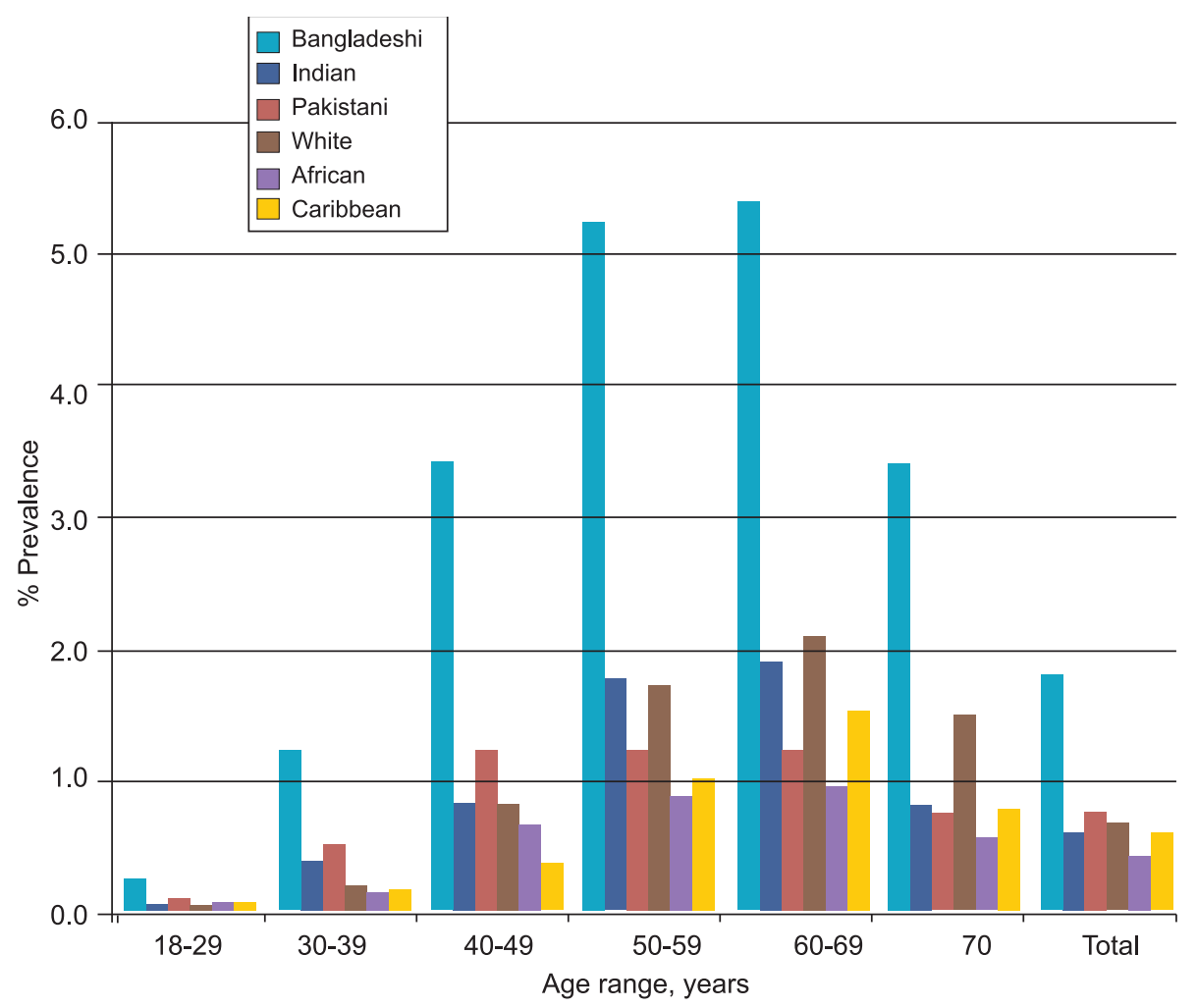

Fig.-5: Showing Prevalence of NAFLD among different ethnic group in different aged group people living in UK.

conservative attitude leading to sedentary life style of the female may be responsible for this. ${ }^{29,} 30$ Affluence is another reason for development of NAFLD. ${ }^{22}$ The child and adolescent populations of urban society with very little physical activity will constitute the future burden of NAFLD in the country in near future. ALT as well as GGT is good detector of NASH in Bangladeshi population with GGT having better sensitivity and specificity. ${ }^{14}$ Liver biopsy is the gold standard for assessing severity of NAFLD and that is safe in expert hand. ${ }^{31}$ Although IR has the central role in the pathogenesis of NAFLD, a subgroup of NAFLD of Bangladeshi population are not associated with IR, where genetic polymorphism and dietary habit may be responsible for it. ${ }^{32}$ The most alarming feature is that there is a high prevalence of NASH of about $40 \%$ among the NAFLD patients ${ }^{14,25,27}$ These NASH patients are the future patients of cirrhosis and hepatocellular carcinoma.

\section{Changing landscape of liver disease of Bangladesh:}

Hepatitis $B$ and hepatitis $C$ have been the leading causes of mortality and morbidity from chronic liver disease in Bangladesh. But with increase in awareness and mass vaccination against $\mathrm{HBV}$, prevalence of both the diseases has been decreasing in the country. - With the changes in socioeconomic condition and life style, the predominant aetiology of chronic liver disease is drifting from infectious to noninfectious diseases and the contribution of NAFLD is progressively increasing.

\section{Conclusion:}

NAFLD is emerging as the largest contributor of chronic liver disease in Bangladesh. Both obese and nonobese populations are affected. Insulin resistance, genetic factors, dietary habit and sedentary life style - all may be responsible for this. This is the time to start "a stitch to save nine" for this new frontier of hepatology to combat the crisis. This warrants the attention of health 
policy makers, stakeholders, service providers, clinicians and social worker to explore this frontier and combat it from right now.

\section{References:}

1. Arab JP, Candia R, Zapata R, Muñoz C, Arancibia JP, Poniachik J, Soza A, Fuster F, Brahm J, Sanhueza E, Contreras C, Cuellar MC, Arrese M, Riquelme A. Management of nonalcoholic fatty liver disease: An evidence-based clinical practice review. World J Gastroenterol 2014;20:12182-12201.

2. Zhang TS, Qin HL, Wang T, Li HT, Li H, Xia SH, Xiang ZH. Global publication trends and research hotspots of nonalcoholic fatty liver disease: a bibliometric analysis and systematic review. Springer Plus 2015; 4:776.

3. Alazawi W, Mathur R, Abeysekera K, Hull S, Boomla K, Robson J, Foster GR. Ethnicity, and the diagnosis gap in liver disease: a population-based study. Br J Gen Pract 2014; 694-702.

4. Chalasani N, Younossi Z, Lavine JE, Diehl AM, Brunt EM, Cusi K, Charlton M, Sanyal AJ. The diagnosis and management of non alcoholic fatty liver disease: practice Guideline by the American Association for the Study of Liver Diseases, American College of Gastroenterology, and the American Gastroenterological Association. Hepatology 2012; 55:2005-2023.

5. Clark JM, Diehl AM. Nonalcoholic fatty liver disease: an underrecognized cause of cryptogenic cirrhosis.JAMA. 2003; 289:3000-4.

6. Ashtari S, Pourhoseingholi MA, Zali MR. Non-alcohol fatty liver disease in Asia: Prevention and planning. World J Hepatol 2015; 7:1788-1796.

7. Chan WK, Tan AT, Vethakkan SR, Tah PC, Vijayananthan A, Goh KL. Low physical activity and energy dense Malaysian foods are associated with non-alcoholic fatty liver disease in centrally obese but not in non-centrally obese patients with diabetes mellitus. Asia Pac J Clin Nutr. 2015; 24:289-98.

8. Amarapurkar DN, Hashimoto E, Lesmana LA, Sollano JD, Chen PJ, Goh KL. How common is non-alcoholic fatty liver disease in the Asia-Pacific region and are there local differences? J Gastroenterol Hepatol. 2007; 22:788-93.

9. Tominaga K, Kurata JH, Chen YK, Fujimoto E, Miyagawa S, Abe I, et al. Prevalence of fatty liver in Japanese children and relationship to obesity. An epidemiological ultrasonographic survey. Dig Dis Sci.1995;40:2002-9.

10. Franzese A, Vajro P, Argenziano A, Puzziello A, Iannucci MP, Saviano MC, et al. Liver involvement in obese children. Ultrasonography and liver enzyme levels at diagnosis and during follow-up in an Italian population. Dig Dis Sci. 1997;42:1428-32.

11. Lam B, Younossi ZM. Treatment options for nonalcoholic fatty liver disease. Ther Adv Gastroenterol. 2010;3(2): 121-137
12. Alam S, Mustafa G, Alam M, Ahmad N. Insulin resistance in development and progression of nonalcoholic fatty liver disease.World J Gastrointest Pathophysiol 2016; 7(2): 211-217

13. Biswas PK, Kabir AKMH, Karim ME, Hossain MS, Vhadury SC, Rhaman MM, Masud H, Khan MR. Incidence and Risk Factors of Non-Alcoholic Fatty Liver Disease among NonObese Patients Attending at Department of Gastroenterology, BSMMU. J MEDICINE 2015; 16: 89-92.

14. Alam S, Noor-E-Alam SM, Chowdhury ZR, Alam M, Kabir J. Nonalcoholic steatohepatitis in nonalcoholic fatty liver disease patients of Bangladesh. World J Hepatol 2013; 5:281-7.

15. Chowdhury A, Younossi ZM. Global Epidemiology and Risk Factors for Nonalcoholic Fatty Liver Disease. Alcoholic and Non-Alcoholic Fatty Liver Disease. Springer International Publishing Switzerland 2016;21-40.

16. Mahady SE, George J. The future liver of the Asia pacific: fatter and firmer from more fructose and fortune? J Clin Exp Hepatol 2013;3:106-13.

17. Rahman M, Abedin T, Amin R, Rahman MR, Faiz MA. Non Alcoholic Fatty Liver Disease- Is It Always Benign? J Bangladesh Coll Phys Surg 2007;25:144-152.

18. Ruhl CE, Everhart JE. Fatty liver indices in the multiethnic United States National Health and Nutrition Examination Survey. Alimentary pharmacology \& therapeutics. 2015; 41: 65-76.

19. Munteanu MA, Nagy GA, Mircea PA. Current Management of NAFLD. Clujul Med 2016; 89:19-23.

20. Parkash O, Hamid S. Are we ready for a new epidemic of under recognized liver disease in South Asia especially in Pakistan? Non alcoholic fatty liver disease. J Pak Med Assoc 2013; 63:95-9.

21. Hoque MI. NAFLD in Bangladesh. Abstract Book 1st Conference of SASL 2013; 69.

22. Rahman MM, Kibria MG, Begum H, Haque M, Sultana N, Akhter M et al. Prevalence and Risk Factors of Nonalcoholic Fatty Liver Disease in a Rural Community of South Asia. Gastroenterology 2015; 148: S1045 - 6 .

23. Ahmed F. Country report of Liver disease in Bangladesh. Abstract Book 1st Conference of SASL 2013; 45.

24. Abbas Z. Country report of Liver disease in Pakistan. Abstract Book 1st Conference of SASL 2013; 45.

25. Dassanayake A. Country report of Liver disease in Srilanka. Abstract Book 1st Conference of SASL 2013; 49.

26. Amarapurkar D, Kamani P, Patel N, Gupte P, Kumar P, Agal $\mathrm{S}$, et al. Prevalence of non-alcoholic fatty liver disease: population based study. Ann Hepatol. 2007;6:161.

27. Alam S, Gupta UD, Alam M, Kabir J, Chowdhury ZR, Alam AKMK. Clinical, anthropometric, biochemical and 
histological characteristics of nonobese nonalcoholic fatty liver disease patients of Bangladesh. Indian J Gastroenterol 2014; 33(5):452-457.

28. Alam S, Mustafa G, Ahmad N,Khan M. .Patients profile of nonalcoholic fatty liver disease of Bangladesh. Hepatology International 2007; 1: 3-242.

29. Alam S, Gupta UD, Kabir J, Alam SMNE,Chowdhury ZR, Alam AKMK. Transaminases and Gamma Glutamyl Transpeptidase for Detecting Nonalcoholic Steatohepatitis and Fibrosis in Nonalcoholic Fatty Liver Disease. .BSMMU J 2015; 8 (1): 61-67.

30. Alam S, Alam M, Alam SMNE, Chowdhury ZR, Kabir J. Prevalence and Predictor of Nonalcoholic Steatohepatitis (NASH) in Nonalcoholic Fatty Liver Disease (NAFLD). J Banagladesh Coll Phys Surg 2014; 32: 71-77.

31. Mustafa G, Alam S, Al Mamun A, Ahmad N, Alam K, Khan M. Per-cutaneous Liver Biopsy: Technique and Safety. Hepatogastroenterology. 2011; 58(106):529-31.
32. Alam S, Das U, Hasan N1, Kabir J,Das DC, Alam AKMK. Insulin resistance in nonalcoholic fatty liver disease revalidation of old concept in the new era. Hepatology International 2015;9: S115.

33. Ahmad N, Alam S, Mustafa G, Chowdhury ZR, Baig RH,Ahmed $M$ et al. Seroprevalence of hepatitis B Virus in Parents and Under 5 Years Children in a Rural Area of Bangladesh. BSMMU J 2011; 4(1): 8-11.

34. Khan M, Mustafa MG, Ahmad N, Alam M, Baig RH, Chowdhry ZR, Ahmed M. Seroprevalence of hepatitis C virus in rural population of Bangladesh.Indian J Gastroenterol. 2010; 29(1):38-9.

35. Alam S, Ahmad N, Khan M, Mustafa G, Mamun AA, Mashud G. Seroprevalence of hepatitis $\mathrm{C}$ virus infection among health care workers. Journal of Bangladesh College of Physicians and Surgeons 2007; 25(3); 126-129. 